\title{
Research Article On Fuzzy Soft Sets
}

\author{
B. Ahmad ${ }^{1,2}$ and Athar Kharal ${ }^{1}$ \\ ${ }^{1}$ Centre for Advanced Studies in Pure and Applied Mathematics, Bahauddin Zakariya University, 60800 Multan, Pakistan \\ ${ }^{2}$ Department of Mathematics, King Abdul Aziz University, P.O. Box 80203, Jeddah 21589, Saudi Arabia
}

Correspondence should be addressed to Athar Kharal, atharkharal@gmail.com

Received 17 November 2008; Revised 28 February 2009; Accepted 5 June 2009

Recommended by Yasar Becerikli

We further contribute to the properties of fuzzy soft sets as defined and studied in the work of Maji et al. ( 2001), Roy and Maji (2007), and Yang et al. (2007) and support them with examples and counterexamples. We improve Proposition 3.3 by Maji et al., (2001) . Finally we define arbitrary fuzzy soft union and fuzzy soft intersection and prove DeMorgan Inclusions and DeMorgan Laws in Fuzzy Soft Set Theory.

Copyright (C) 2009 B. Ahmad and A. Kharal. This is an open access article distributed under the Creative Commons Attribution License, which permits unrestricted use, distribution, and reproduction in any medium, provided the original work is properly cited.

\section{Introduction}

In 1999, Molodtsov [1] introduced soft sets and established the fundamental results of the new theory. It is a general mathematical tool for dealing with objects which have been defined using a very loose and hence very general set of characteristics. A soft set is a collection of approximate descriptions of an object. Each approximate description has two parts: a predicate and an approximate value set. In classical mathematics, we construct a mathematical model of an object and define the notion of the exact solution of this model. Usually the mathematical model is too complicated and we cannot find the exact solution. So, in the second step, we introduce the notion of approximate solution and calculate that solution. In the Soft Set Theory (SST), we have the opposite approach to this problem. The initial description of the object has an approximate nature, and we do not need to introduce the notion of exact solution. The absence of any restrictions on the approximate description in SST makes this theory very convenient and easily applicable in practice. We can use any parametrization we prefer with the help of words and sentences, real numbers, functions, mappings, and so on. It means that the problem of setting the membership function or any similar problem does not arise in SST. In [1], besides demarcating the basic contours of SST, Molodtsov also showed how SST is free from parametrization inadequacy syndrom of Fuzzy Set Theory (FST), Rough Set Theory (RST),Probability Theory, and
Game Theory. SST is a very general framework. Many of the established paradigms appear as special cases of SST.

Applications of Soft Set Theory in other disciplines and real life problems are now catching momentum. Molodtsov [1] successfully applied the soft theory into several directions, such as smoothness of functions, game theory, operations research, Riemann integration, Perron integration, theory of probability, theory of measurement, and so on. Maji et al. [2] gave first practical application of soft sets in decision making problems. It is based on the notion of knowledge reduction in rough set theory. Maji et al. [3] defined and studied several basic notions of soft set theory in 2003. In 2005, Pei and Miao [4] and Chen et al. [5] improved the work of Maji et al. [2, 3].

Many researchers have contributed towards the fuzzification of the notion of soft set, for example, [6-8]. In this paper, we present some more properties of fuzzy soft union and fuzzy soft intersection as defined by Maji et al. [6], and support them by examples and counterexamples. We also revise Maji's definition of fuzzy soft intersection and improve [6, Proposition 3.3]. Finally we define arbitrary fuzzy soft union and intersection and prove DeMorgan Inclusions and DeMorgan Laws in Fuzzy Soft Set Theory.

\section{Basic Definitions Revisited}

Throughout this paper, $X$ refers to an initial universe, $E$ is a set of parameters, $\Sigma, \Omega \subseteq E$, and $\widetilde{P}(X)$ is the set of all fuzzy sets of $X$. 
Maji et al. defined a fuzzy soft set in the following manner.

Definition 1 (see [6]). A pair $(\Lambda, \Sigma)$ is called a fuzzy soft set over $X$, where $\Lambda: \Sigma \rightarrow \widetilde{P}(X)$ is a mapping from $\Sigma$ into $\widetilde{P}(X)$.

Definition 2. Let $X$ be a universe and $E$ a set of attributes. Then the pair $\widetilde{(X, E)}$ denotes the collection of all fuzzy soft sets on $X$ with attributes from $E$ and is called a fuzzy soft class.

Definition 3 (see [6]). For two fuzzy soft sets $(\Lambda, \Sigma)$ and $(\Delta, \Omega)$ in a fuzzy soft class $\widehat{(X, E)}$, we say that $(\Lambda, \Sigma)$ is a fuzzy soft subset of $(\Delta, \Omega)$, if

(i) $\Sigma \subseteq \Omega$,

(ii) For all $\varepsilon \in \Sigma, \Lambda(\varepsilon) \leq \Delta(\varepsilon)$,

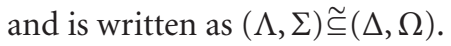

Definition 4 (see [6]). The complement of a fuzzy soft set $(\Lambda, \Sigma)$ is denoted by $(\Lambda, \Sigma)^{c}$ and is defined by $(\Lambda, \Sigma)^{c}=$ $\left.\left(\Lambda^{c},\right\rceil \Sigma\right)$, where $\left.\Lambda^{c}:\right\rceil \Sigma \rightarrow \widetilde{P}(X)$ is a mapping given by $\Lambda^{c}(\sigma)=(\Lambda(\neg \sigma))^{c}$, for all $\left.\sigma\right\rceil \in \Sigma$.

Union of two fuzzy soft sets is defined by Maji et al. [6] as follows.

Definition 5 (see [6]). Union of two fuzzy soft sets $(\Lambda, \Sigma)$ and $(\Delta, \Omega)$ in a soft class $(X, E)$ is a fuzzy soft set $(\Theta, \Xi)$, where $\Xi=\Sigma \cup \Omega$, and for all $\varepsilon \in \Xi$,

$$
\Theta(\varepsilon)= \begin{cases}\Lambda(\varepsilon), & \text { if } \varepsilon \in \Sigma-\Omega, \\ \Delta(\varepsilon), & \text { if } \varepsilon \in \Omega-\Sigma, \\ \Lambda(\varepsilon) \vee \Delta(\varepsilon), & \text { if } \varepsilon \in \Sigma \cap \Omega,\end{cases}
$$

and is written as $(\Lambda, \Sigma) \tilde{V}(\Delta, \Omega)=(\Theta, \Xi)$.

For a few basic properties of fuzzy soft union, we refer to [6, Proposition 3.2]. Moreover, we have some more properties.

Proposition 1. Let $(\Lambda, \Sigma),(\Delta, \Omega)$, and $(\Theta, \Xi)$ be fuzzy soft sets in $\overline{(X, E)}$. Then one has the following:

(1) $(\Lambda, \Sigma) \tilde{V}(\Delta, \Omega)=(\Delta, \Omega) \tilde{V}(\Lambda, \Sigma)$,

(2) $(\Lambda, \Sigma) \tilde{V}((\Delta, \Omega) \tilde{\bigvee}(\Theta, \Xi))=((\Lambda, \Sigma) \tilde{\bigvee}(\Delta, \Omega)) \tilde{V}(\Theta, \Xi)$,

(3) $(\Lambda, \Sigma) \tilde{\subseteq}(\Lambda, \Sigma) \tilde{\bigvee}(\Delta, \Omega)$ and $(\Delta, \Omega) \tilde{\subseteq}(\Lambda, \Sigma) \widetilde{V}(\Delta, \Omega)$,

(4) $(\Lambda, \Sigma) \widetilde{\subseteq}(\Delta, \Omega) \Rightarrow(\Lambda, \Sigma) \tilde{V}(\Delta, \Omega)=(\Delta, \Omega)$,

Maji et al. defined the intersection of two fuzzy soft sets as follows.

Definition 6 (see [6]). Intersection of two fuzzy soft sets $(\Lambda, \Sigma)$ and $(\Delta, \Omega)$ in a fuzzy soft class $\widehat{(X, E)}$ is a fuzzy soft set $(\Theta, \Xi)$, where $\Xi=\Sigma \cap \Omega$, and for all $\varepsilon \in \Xi, \Theta(\varepsilon)=$ $\Lambda(\varepsilon)$ or $\Delta(\varepsilon)$ (as both are same fuzzy set), and is written as $(\Lambda, \Sigma) \tilde{\Lambda}(\Delta, \Omega)=(\Theta, \Xi)$.

We point out that generally $\Lambda(\varepsilon)$ and $\Delta(\varepsilon)$ may not be identical. Moreover, $\Sigma \cap \Omega$ must be nonempty to avoid the degenerate case. Thus we revise Definition 6 as follows.

Definition 7. Let $(\Lambda, \Sigma)$ and $(\Delta, \Omega)$ be two fuzzy soft sets in a fuzzy soft class $\widehat{(X, E)}$ with $\Sigma \cap \Omega \neq \phi$. Then intersection of two fuzzy soft sets $(\Lambda, \Sigma)$ and $(\Delta, \Omega)$ is a fuzzy soft set $(\Theta, \Xi)$, where $\Xi=\Sigma \cap \Omega$, and for all $\varepsilon \in \Xi, \Theta(\varepsilon)=\Lambda(\varepsilon) \wedge \Delta(\varepsilon)$. We write $(\Lambda, \Sigma) \tilde{\Lambda}(\Delta, \Omega)=(\Theta, \Xi)$.

The following example explains Definition 7.

Example 1. Suppose that

$$
\begin{aligned}
X & =\{h, i, j, k\}, \\
E & =\{\text { very costly, costly, beautiful, } \\
& \text { in the green surroundings, cheap }\} .
\end{aligned}
$$

Consider the soft set $(\Lambda, \Sigma)$ which describes the "cost of the houses" and the soft set $(\Delta, \Omega)$ which describes the "attractiveness of the houses." Thus we take $\Sigma, \Delta \subseteq E$ as

$\Sigma=\{$ very costly, costly, cheap $\}$,

$\Delta=\{$ beautiful, in the green surroundings, cheap $\}$.

and suppose that

$$
\begin{gathered}
\Lambda(\text { very costly })=\left\{h_{0.3}, i_{0.4}, j_{0.1}, k_{0.8}\right\}, \\
\Lambda(\text { costly })=\left\{h_{0.5}, i_{0.2}, j_{0.7}, k_{1.0}\right\}, \\
\Lambda(\text { cheap })=\left\{h_{0.3}, i_{0.1}, j_{0.8}, k_{0.9}\right\} \\
\Delta(\text { beautiful })=\left\{h_{0.4}, i_{0.7}, j_{0.2}, k_{0.1}\right\},
\end{gathered}
$$

$\Delta$ (in the green surroundings $)=\left\{h_{0.9}, i_{0.3}, j_{0.4}, k_{0.6}\right\}$,

$$
\Delta(\text { cheap })=\left\{h_{0.5}, i_{0.6}, j_{0.2}, k_{0.5}\right\} .
$$

Then $(\Lambda, \Sigma) \tilde{\Lambda}(\Delta, \Omega)=(\Theta, \Xi)$ where $\Xi=\Sigma \cap \Omega=\{$ cheap $\}$. Now if we use the definition of Maji et al., we get two different values for $\Theta$ (cheap), that is,

$$
\begin{aligned}
\Theta(\text { cheap }) & =\Lambda(\text { cheap }) \\
& =\left\{h_{0.3}, i_{0.1}, j_{0.8}, k_{0.9}\right\} \\
& \neq\left\{h_{0.5}, i_{0.6}, j_{0.2}, k_{0.5}\right\} \\
& =\Delta(\text { cheap }) .
\end{aligned}
$$

Therefore, by using [6, Definition 7], $\Theta$ ceases to be a function as $\Lambda$ (cheap) and $\Delta$ (cheap) are not identical and so this definition is not applicable. However by using Definition 7, we have

$$
\Theta(\text { cheap })=\Lambda(\text { cheap }) \wedge \Delta(\text { cheap })=\left\{h_{0.3}, i_{0.1}, j_{0.2}, k_{0.5}\right\} .
$$


For some basic properties of fuzzy soft intersection, we refer to [6, Proposition 3.2]. Moreover, we have some more properties:

Proposition 2. Let $(\Lambda, \Sigma),(\Delta, \Omega)$, and $(\Theta, \Xi)$ be fuzzy soft sets in a fuzzy soft class $(X, E)$. Then one has following:

(1) $(\Lambda, \Sigma) \tilde{\Lambda}(\Lambda, \Sigma)=(\Lambda, \Sigma)$,

(2) $(\Lambda, \Sigma) \tilde{\Lambda}(\Delta, \Omega)=(\Delta, \Omega) \tilde{\Lambda}(\Lambda, \Sigma)$,

(3) $(\Lambda, \Sigma) \tilde{\Lambda}(\Delta, \Omega) \widetilde{\subseteq}(\Lambda, \Sigma)$ and $(\Lambda, \Sigma) \tilde{\Lambda}(\Delta, \Omega) \widetilde{\subseteq}(\Delta, \Omega)$,

(4) $(\Lambda, \Sigma) \widetilde{\simeq}(\Delta, \Omega) \Rightarrow(\Lambda, \Sigma) \tilde{\Lambda}(\Delta, \Omega)=(\Lambda, \Sigma)$,

(5) $((\Lambda, \Sigma) \tilde{\Lambda}(\Delta, \Omega)) \tilde{\Lambda}(\Theta, \Xi)=(\Lambda, \Sigma) \tilde{\Lambda}((\Delta, \Omega) \tilde{\Lambda}(\Theta, \Xi))$.

In [6, Definition 3.6], it is shown that

$$
\tilde{\Sigma}^{c}=\Phi, \quad \Phi^{c}=\tilde{\Sigma} .
$$

The following example shows that these do not hold in general.

Example 2. Let $\widetilde{(X, E)}$ be a fuzzy soft class, where

$$
X=\{a, b, c\}, \quad E=\left\{e_{1}, e_{2}, e_{3}, e_{4}\right\},
$$

choose $\Sigma=\left\{e_{1}, e_{3}\right\}$, and

$$
\begin{aligned}
\widetilde{\Sigma} & =\left\{e_{1}=\left\{a_{1}, b_{1}, c_{1}\right\}, e_{3}=\left\{a_{1}, b_{1}, c_{1}\right\}\right\} \\
\Phi & =\left\{e_{1}=\left\{a_{0}, b_{0}, c_{0}\right\}, e_{3}=\left\{a_{0}, b_{0}, c_{0}\right\}\right\} .
\end{aligned}
$$

Calculations give

$$
\begin{gathered}
\Phi^{c}=\left\{\neg e_{1}=\left\{a_{1}, b_{1}, c_{1}\right\}, \neg e_{3}=\left\{a_{1}, b_{1}, c_{1}\right\}\right\} \\
\neq\left\{e_{1}=\left\{a_{1}, b_{1}, c_{1}\right\}, e_{3}=\left\{a_{1}, b_{1}, c_{1}\right\}\right\}=\widetilde{\Sigma}, \\
\tilde{\Sigma}^{c}=\left\{\neg e_{1}=\left\{a_{0}, b_{0}, c_{0}\right\}, \neg e_{3}=\left\{a_{0}, b_{0}, c_{0}\right\}\right\} \\
\quad \neq\left\{e_{1}=\left\{a_{0}, b_{0}, c_{0}\right\}, e_{3}=\left\{a_{0}, b_{0}, c_{0}\right\}\right\}=\Phi .
\end{gathered}
$$

\section{DeMorgan Inclusions and Laws}

Maji et al. proved the following Proposition.

Proposition 3 (see [6, Proposition 3.3]). It holds that

(1) $[(\Lambda, \Sigma) \tilde{V}(\Delta, \Omega)]^{c}=(\Lambda, \Sigma)^{c} \tilde{V}(\Delta, \Omega)^{c}$,

(2) $[(\Lambda, \Sigma) \tilde{\Lambda}(\Delta, \Omega)]^{c}=(\Lambda, \Sigma)^{c} \tilde{\Lambda}(\Delta, \Omega)^{c}$.

The following example shows that (14) and (16) of Proposition 3 do not hold.

Example 3. Let $X=\{a, b, c\}$ and $E=\left\{e_{1}, e_{2}, e_{3}, e_{4}\right\}$ and $(\Lambda, \Sigma)$ and $(\Delta, \Omega)$ fuzzy soft sets in a fuzzy soft class $\widetilde{(X, E)}$ given as

$$
\begin{aligned}
& (\Lambda, \Sigma)=\left\{e_{3}=\left\{a_{0.4}, b_{0.6}, c_{0.7}\right\}, e_{4}=\left\{a_{0.4}, b_{0.3}, c_{1}\right\}\right\}, \\
& (\Delta, \Omega)=\left\{e_{2}=\left\{a_{0.2}, b_{0.2}, c_{0.7}\right\}, e_{4}=\left\{a_{0.7}, b_{0.8}, c_{0.6}\right\}\right\} .
\end{aligned}
$$

Then calculations show that

$$
\begin{array}{r}
{[(\Lambda, \Sigma) \widetilde{\bigvee}(\Delta, \Omega)]^{c}=\left\{\neg e_{2}=\left\{a_{0.8}, b_{0.8}, c_{0.3}\right\},\right.} \\
\neg e_{3}=\left\{a_{0.6}, b_{0.4}, c_{0.3}\right\}, \\
\left.\neg e_{4}=\left\{a_{0.3}, b_{0.2}, c_{0}\right\}\right\} \\
\neq\left\{\neg e_{2}=\left\{a_{0.8}, b_{0.8}, c_{0.3}\right\},\right. \\
\neg e_{3}=\left\{a_{0.6}, b_{0.4}, c_{0.3}\right\}, \\
\left.\neg e_{4}=\left\{a_{0.6}, b_{0.7}, c_{0.4}\right\}\right\} \\
=(\Lambda, \Sigma)^{c} \widetilde{\bigvee}(\Delta, \Omega)^{c}, \\
{[(\Lambda, \Sigma) \widetilde{\Lambda}(\Delta, \Omega)]^{c}=\left\{\neg e_{4}=\left\{a_{0.6}, b_{0.7}, c_{0.4}\right\}\right\}} \\
\neq\left\{\neg e_{4}=\left\{a_{0.3}, b_{0.2}, c_{0}\right\}\right\} \\
=(\Lambda, \Sigma)^{c} \widetilde{\wedge}(\Delta, \Omega)^{c} .
\end{array}
$$

However, we partially establish identities (14) and (16) of [6, Proposition 3.3] as follows.

Theorem 1. For fuzzy soft sets $(\Lambda, \Sigma)$ and $(\Delta, \Omega)$ in $\widetilde{(X, E)}$, one has the following:

(1) $[(\Lambda, \Sigma) \tilde{V}(\Delta, \Omega)]^{c} \widetilde{\simeq}(\Lambda, \Sigma)^{c} \tilde{V}(\Delta, \Omega)^{c}$,

(2) $(\Lambda, \Sigma)^{c} \tilde{\Lambda}(\Delta, \Omega)^{c} \widetilde{\subseteq}[(\Lambda, \Sigma) \tilde{\Lambda}(\Delta, \Omega)]^{c}$.

Proof. (1) Suppose that $(\Lambda, \Sigma) \tilde{\bigvee}(\Delta, \Omega)=(\Theta, \Sigma \cup \Omega)$. Therefore,

$$
\begin{aligned}
{[(\Lambda, \Sigma) \widetilde{V}(\Delta, \Omega)]^{c} } & =(\Theta, \Sigma \cup \Omega)^{c} \\
& \left.\left.\left.=\left(\Theta^{c},\right\rceil(\Sigma \cup \Omega)\right)=\left(\Theta^{c},\right\rceil \Sigma \cup\right\rceil \Omega\right)
\end{aligned}
$$

by [6, Proposition 2.1]. For $\neg \alpha \in\rceil \Sigma \cup\rceil \Omega$

$$
\begin{aligned}
\Theta^{c}(\neg \alpha)= & {[\Theta(\alpha)]^{c} } \\
= & \begin{cases}{[\Lambda(\alpha)]^{c},} & \text { if } \neg \alpha \in\rceil \Sigma-\rceil \Omega, \\
{[\Delta(\alpha)]^{c},} & \text { if } \neg \alpha \in\rceil \Omega-\rceil \Sigma, \\
{[\Lambda(\alpha) \vee \Delta(\alpha)]^{c},} & \text { if } \neg \alpha \in\rceil \Sigma \cap\rceil \Omega,\end{cases} \\
= & \begin{cases}\Lambda^{c}(\neg \alpha), & \text { if } \neg \alpha \in\rceil \Sigma-\rceil \Omega, \\
\Delta^{c}(\neg \alpha), & \text { if } \neg \alpha \in\rceil \Omega-\rceil \Sigma, \\
\Lambda^{c}(\neg \alpha) \wedge \Delta^{c}(\neg \alpha), & \text { if } \neg \alpha \in\rceil \Sigma \cap\rceil \Omega .\end{cases}
\end{aligned}
$$

Now consider

$$
\begin{aligned}
(\Delta, \Omega)^{c} \widetilde{\bigvee}(\Delta, \Omega)^{c} & \left.\left.=\left(\Lambda^{c},\right\rceil \Sigma\right) \widetilde{\bigvee}\left(\Delta^{c},\right\rceil \Omega\right) \\
& =(\Gamma,\rceil \Sigma \cap\rceil \Omega), \quad(\text { say }),
\end{aligned}
$$


where

$$
\Gamma(\neg \alpha)= \begin{cases}\Lambda^{c}(\neg \alpha), & \text { if } \neg \alpha \in\rceil \Sigma-\rceil \Omega, \\ \Delta^{c}(\neg \alpha), & \text { if } \neg \alpha \in\rceil \Omega-\rceil \Sigma, \\ \Lambda^{c}(\neg \alpha) \vee \Delta^{c}(\neg \alpha), & \text { if } \neg \alpha \in \neg \alpha \in\rceil \Sigma \cap\rceil \Sigma .\end{cases}
$$

From (14) and (16), we get (1).

(2) Consider $\left.\left.(\Lambda, \Sigma)^{c} \tilde{\Lambda}(\Delta, \Omega)^{c}=\left(\Lambda^{c},\right\rceil \Sigma\right) \tilde{V}\left(\Delta^{c},\right\rceil \Omega\right)=$ $(\Theta,\rceil \Sigma \cap\rceil \Omega)$ (say), where

$$
\left.\left.\Theta(\neg \alpha)=\Lambda^{c}(\neg \alpha) \wedge \Delta^{c}(\neg \alpha), \quad \forall \neg \alpha \in\right\rceil \Sigma \cap\right\rceil \Omega .
$$

On the other hand

$$
\begin{aligned}
{[(\Lambda, \Sigma) \widetilde{\Lambda}(\Delta, \Omega)]^{c} } & =(\Gamma, \Sigma \cap \Omega)^{c} \quad(\text { say }) \\
& \left.\left.=\left(\Gamma^{c},\right\rceil \Sigma \cap\right\rceil \Omega\right)
\end{aligned}
$$

Now for $\neg \alpha \in] \Sigma \cap] \Omega$

$$
\begin{aligned}
\Gamma^{c}(\neg \alpha) & =[\Gamma(\alpha)]^{c} \\
& =[\Lambda(\alpha) \wedge \Delta(\alpha)]^{c} \\
& =\Lambda^{c}(\neg \alpha) \vee \Delta^{c}(\neg \alpha) .
\end{aligned}
$$

Clearly,

$$
\Theta(\neg \alpha)=\Lambda^{c}(\neg \alpha) \wedge \Delta^{c}(\neg \alpha) \subseteq \Lambda^{c}(\neg \alpha) \vee \Delta^{c}(\neg \alpha)=\Gamma^{c}(\neg \alpha) .
$$

hence is the result.

In general, these inclusions cannot be reversed, as is evident from Example 3.

It is well known that DeMorgan Laws interrelate union and intersection via complements. Here first, we prove the following DeMorgan Inclusions.

Theorem 2. For soft sets $(\Lambda, \Sigma)$ and $(\Delta, \Omega)$ of a soft class $(X, E)$, one has the following.

$$
\begin{aligned}
& \text { (1) }(\Lambda, \Sigma)^{c} \tilde{\Lambda}(\Delta, \Omega)^{c} \widetilde{\subseteq}[(\Lambda, \Sigma) \tilde{V}(\Delta, \Omega)]^{c}, \\
& \text { (2) }[(\Lambda, \Sigma) \tilde{\Lambda}(\Delta, \Omega)]^{c} \widetilde{\subseteq}(\Lambda, \Sigma)^{c} \tilde{V}(\Delta, \Omega)^{c} .
\end{aligned}
$$

Proof. (1)Consider

$$
\begin{aligned}
(\Lambda, \Sigma)^{c} \widetilde{\Lambda}(\Delta, \Omega)^{c} & \left.\left.=\left(\Lambda^{c},\right\rceil \Sigma\right) \widetilde{\Lambda}\left(\Delta^{c},\right\rceil \Omega\right) \\
& =(\Theta,\rceil \Sigma \cap\rceil \Omega), \quad \text { (say) },
\end{aligned}
$$

where

$$
\Theta(\neg \alpha)=\Lambda^{c}(\neg \alpha) \wedge \Delta^{c}(\neg \alpha),
$$

$\neg \alpha \in \Sigma \cap \Omega$. fore,

Again suppose that $(\Lambda, \Sigma) \tilde{V}(\Delta, \Omega)=(\Gamma, \Sigma \cup \Omega)$. There-

$$
\begin{aligned}
((\Lambda, \Sigma) \widetilde{\bigvee}(\Delta, \Omega))^{c} & =(\Gamma, \Sigma \cup \Omega)^{c} \\
& \left.\left.=\left(\Gamma^{c},\right\rceil \Sigma \cap\right\rceil \Omega\right)=\left(\Gamma^{c}, \Sigma \cup \Omega\right)
\end{aligned}
$$

by [6, Proposition 2.1]. For $\neg \alpha \in \Sigma \cup \Omega$, we have

$$
\Gamma^{c}(\neg \alpha)=[\Gamma(\alpha)]^{c}= \begin{cases}{[\Lambda(\alpha)]^{c},} & \alpha \in\rceil \Sigma-\rceil \Omega, \\ {[\Delta(\alpha)]^{c},} & \alpha \in\rceil \Omega-\rceil \Sigma, \\ {[\Lambda(\alpha) \vee \Delta(\alpha)]^{c},} & \alpha \in\rceil \Sigma \cap\rceil \Omega .\end{cases}
$$

For $\neg \alpha \in\rceil \Sigma \cap\rceil \Omega$, we have

$$
\Gamma^{c}(\neg \alpha)=[\Lambda(\alpha) \vee \Delta(\alpha)]^{c}=\Lambda^{c}(\neg \alpha) \wedge \Delta^{c}(\neg \alpha) .
$$

From (22) and (24), we get (1).

(2) Suppose that $(\Lambda, \Sigma) \tilde{\Lambda}(\Delta, \Omega)=(\Theta, \Sigma \cap \Omega)$, where

$$
\Theta(\alpha)=\Lambda(\alpha) \wedge \Delta(\alpha), \quad \text { for } \alpha \in \Sigma \cap \Omega
$$

Therefore,

$$
\begin{aligned}
{[(\Lambda, \Sigma) \widetilde{\Lambda}(\Delta, \Omega)]^{c} } & =(\Theta, \Sigma \cap \Omega)^{c} \\
& \left.\left.\left.=\left(\Theta^{c},\right\rceil(\Sigma \cap \Omega)\right)=\left(\Theta^{c},\right\rceil \Sigma \cap\right\rceil \Omega\right)
\end{aligned}
$$

by [6, Proposition 2.1]. Now take $\neg \alpha \in\rceil \Sigma \cap\rceil \Omega$, then

$$
\begin{aligned}
\Theta^{c}(\neg \alpha) & =[\Theta(\alpha)]^{c}=[\Lambda(\alpha) \wedge \Delta(\alpha)]^{c} \\
& =[\Lambda(\alpha)]^{c} \vee[\Delta(\alpha)]^{c} . \\
\Theta^{c}(\neg \alpha) & =\Lambda^{c}(\neg \alpha) \vee \Delta^{c}(\neg \alpha) .
\end{aligned}
$$

Now consider

$$
\begin{aligned}
(\Lambda, \Sigma)^{c} \widetilde{\bigvee}(\Delta, \Omega)^{c} & \left.\left.=\left(\Lambda^{c},\right\rceil \Sigma\right) \widetilde{\bigvee}\left(\Delta^{c},\right\rceil \Omega\right) \\
& =(\Gamma,\rceil \Sigma \cap\rceil \Omega) \quad(\text { say }) .
\end{aligned}
$$

For $\neg \alpha \in\rceil \Sigma \cup\rceil \Omega$, we have

$$
\Gamma(\neg \alpha)= \begin{cases}\Lambda^{c}(\neg \alpha), & \neg \alpha \in\rceil \Sigma-\rceil \Omega, \\ \Delta^{c}(\neg \alpha), & \neg \alpha \in\rceil \Omega-\rceil \Sigma, \\ \Lambda^{c}(\neg \alpha) \vee \Delta^{c}(\neg \alpha), & \neg \alpha \in\rceil \Sigma \cap\rceil \Omega .\end{cases}
$$

From (28) and (30), we get (2).

The above DeMorgan Inclusions are, in general, irreversible, as is shown in the following.

Example 4. Let $X=\{a, b, c\}$ and $E=\left\{e_{1}, e_{2}, e_{3}, e_{4}\right\}$ and $(\Lambda, \Sigma)$ and $(\Delta, \Omega)$ fuzzy soft sets in a fuzzy soft class $\widehat{(X, E)}$ given as

$$
\begin{gathered}
(\Lambda, \Sigma)=\left\{e_{1}=\left\{a_{0.2}, b_{0}, c_{0.1}\right\}, e_{2}=\left\{a_{0.1}, b_{1}, c_{0.4}\right\},\right. \\
\left.e_{4}=\left\{a_{0.4}, b_{0.7}, c_{1}\right\}\right\}, \\
(\Delta, \Omega)=\left\{e_{2}=\left\{a_{0.9}, b_{0.2}, c_{0.6}\right\}, e_{4}=\left\{a_{0.9}, b_{0.4}, c_{0.7}\right\}\right\} .
\end{gathered}
$$


Then calculations show that

$$
\begin{aligned}
{[(\Lambda, \Sigma) \widetilde{\bigvee}(\Delta, \Omega)]^{c}=} & \left\{\neg e_{1}=\left\{a_{0.8}, b_{1}, c_{0.9}\right\},\right. \\
& \neg e_{2}=\left\{a_{0.1}, b_{0}, c_{0.4}\right\}, \\
& \left.\neg e_{4}=\left\{a_{0.1}, b_{0.3}, c_{0}\right\}\right\} \\
\nsubseteq & \left\{\neg e_{2}=\left\{a_{0.1}, b_{0}, c_{0.4}\right\},\right. \\
& \left.\neg e_{4}=\left\{a_{0.1}, b_{0.3}, c_{0}\right\}\right\} \\
= & (\Lambda, \Sigma)^{c} \widetilde{\wedge}(\Delta, \Omega)^{c}, \\
(\Lambda, \Sigma)^{c} \widetilde{\bigvee}(\Delta, \Omega)^{c}= & \left\{\neg e_{1}=\left\{a_{0.8}, b_{1}, c_{0.9}\right\},\right. \\
& \neg e_{2}=\left\{a_{0.9}, b_{0.8}, c_{0.6}\right\}, \\
& \left.\neg e_{4}=\left\{a_{0.6}, b_{0.6}, c_{0.3}\right\}\right\} \\
\nsubseteq & \left\{\neg e_{2}=\left\{a_{0.9}, b_{0.8}, c_{0.6}\right\},\right. \\
& \left.\neg e_{4}=\left\{a_{0.6}, b_{0.6}, c_{0.3}\right\}\right\} \\
= & {[(\Lambda, \Sigma) \widetilde{\bigwedge}(\Delta, \Omega)]^{c} . }
\end{aligned}
$$

It is natural to ask when the DeMorgan Inclusions in Theorem 2 beome DeMorgan Laws. This is answered in the following.

Theorem 3. For the fuzzy soft sets $(\Lambda, \Sigma)$ and $(\Delta, \Sigma)$ in a fuzzy soft class $\widetilde{(X, E)}$, one has the following:

$$
\begin{aligned}
& \text { (1) }(\Lambda, \Sigma)^{c} \tilde{\Lambda}(\Delta, \Sigma)^{c}=[(\Lambda, \Sigma) \tilde{V}(\Delta, \Sigma)]^{c}, \\
& \text { (2) }[(\Lambda, \Sigma) \tilde{\Lambda}(\Delta, \Sigma)]^{c}=(\Lambda, \Sigma)^{c} \tilde{\bigvee}(\Delta, \Sigma)^{c} .
\end{aligned}
$$

Proof. (1) Consider

$$
\begin{aligned}
(\Lambda, \Sigma)^{c} \widetilde{\Lambda}(\Delta, \Sigma)^{c} & \left.\left.=\left(\Lambda^{c},\right\rceil \Sigma\right) \widetilde{\bigwedge}\left(\Delta^{c},\right\rceil \Sigma\right) \\
& =(\Theta,\rceil \Sigma), \quad \text { (say) }
\end{aligned}
$$

where for all $\neg \alpha \in\rceil \Sigma$

$$
\Theta(\neg \alpha)=\Lambda^{c}(\neg \alpha) \wedge \Delta^{c}(\neg \alpha) .
$$

Again suppose that $(\Lambda, \Sigma) \tilde{V}(\Delta, \Sigma)=(\Gamma, \Sigma)$. Therefore,

$$
\begin{aligned}
((\Lambda, \Sigma) \widetilde{V}(\Delta, \Sigma))^{c} & =(\Gamma, \Sigma)^{c} \\
& \left.=\left(\Gamma^{c},\right\rceil \Sigma\right),
\end{aligned}
$$

where

$$
\Gamma^{c}(\neg \alpha)=[\Gamma(\alpha)]^{c}=[\Lambda(\alpha) \vee \Delta(\alpha)]^{c} .
$$

For all $\neg \alpha \in\rceil \Sigma$, we have

$$
\Gamma^{c}(\neg \alpha)=[\Lambda(\alpha) \vee \Delta(\alpha)]^{c}=\Lambda^{c}(\neg \alpha) \wedge \Delta^{c}(\neg \alpha) .
$$

From (34) and (37), we obtain (2).

(2) Similar to (1).

\section{Generalized DeMorgan Inclusions and Laws}

First, we define arbitrary union and arbitrary intersection of a family of fuzzy soft sets in a fuzzy soft class $\widetilde{(X, E)}$ as follows.

Definition 8. Let $\mathbb{F}=\left\{\left(\Lambda_{i}, \Sigma_{i}\right) \mid i \in I\right\}$ be a family of fuzzy soft

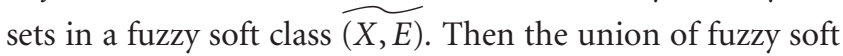
sets in $\mathbb{F}$ is a fuzzy soft set $(\Theta, \Xi), \Xi=\bigcup_{i} \Sigma_{i}$ and for all $\varepsilon \in \Xi$,

$$
\Theta(\varepsilon)=\bigvee_{i} \Delta_{i}\left(\varepsilon, \Sigma_{i}\right)
$$

where

$$
\Delta_{i}\left(\varepsilon, \Sigma_{i}\right)= \begin{cases}\Lambda_{i}(\varepsilon), & \text { if } \varepsilon \in \Sigma_{i}, \\ \Phi, & \text { if } \varepsilon \notin \Sigma_{i} .\end{cases}
$$

The union of three fuzzy soft sets is illustrated as under follows.

Example 5. Let $\widetilde{(X, E)}$ be a fuzzy soft class and $\left(\Lambda_{1}, \Sigma_{1}\right),\left(\Lambda_{2}, \Sigma_{2}\right)$, and $\left(\Lambda_{3}, \Sigma_{3}\right)$, fuzzy soft sets given as

$$
\begin{gathered}
\left(\Lambda_{1}, \Sigma_{1}\right)\left\{e_{1}=\left\{a_{0.9}, b_{0.2}, c_{0.2}\right\}, e_{3}=\left\{a_{0.4}, b_{0.1}, c_{0.7}\right\},\right. \\
\left.e_{4}=\left\{a_{0.3}, b_{0.2}, c_{0}\right\}\right\} \\
\left(\Lambda_{2}, \Sigma_{2}\right)=\left\{e_{1}=\left\{a_{0.4}, b_{0.6}, c_{0.8}\right\}, e_{4}=\left\{a_{0}, b_{0.9}, c_{0.1}\right\}\right\}, \\
\left(\Lambda_{3}, \Sigma_{3}\right)=\left\{e_{1}=\left\{a_{0.8}, b_{0.4}, c_{0.3}\right\}, e_{2}=\left\{a_{0.6}, b_{0.7}, c_{0.9}\right\}\right\} .
\end{gathered}
$$

Calculations give

$$
\begin{aligned}
\left(\Lambda_{1}, \Sigma_{1}\right) \widetilde{V}\left(\Lambda_{2}, \Sigma_{2}\right) \widetilde{V}\left(\Lambda_{3}, \Sigma_{3}\right)=\left\{e_{1}\right. & =\left\{a_{0.9}, b_{0.6}, c_{0.8}\right\}, \\
e_{2} & =\left\{a_{0.6}, b_{0.7}, c_{0.9}\right\}, \\
e_{3} & =\left\{a_{0.4}, b_{0.1}, c_{0.7}\right\}, \\
e_{4} & \left.=\left\{a_{0.3}, b_{0.9}, c_{0.1}\right\}\right\} .
\end{aligned}
$$

Now, we generalize Definition 7 as follows.

Definition 9. Let $\mathbb{F}=\left\{\left(\Lambda_{i}, \Sigma_{i}\right) \mid i \in I\right\}$ be a family of fuzzy soft sets in a fuzzy soft class $\widehat{(X, E)}$, with $\bigcap_{i} \Sigma_{i} \neq \phi$. Then the intersection of fuzzy soft sets in $\mathbb{F}$ is a fuzzy soft set $(\Theta, \Xi)$, where $\Xi=\bigcap_{i} \Sigma_{i}$ and for all $\varepsilon \in \Xi$,

$$
\Theta(\varepsilon)=\bigwedge_{i} \Lambda_{i}(\varepsilon)
$$

We may now generalize Theorem 2 .

Theorem 4. Let $\mathbb{S}=\left\{\left(\Lambda_{i}, \Sigma_{i}\right) \mid i \in I\right\}$ be a family of fuzzy soft sets in a fuzzy soft class $(X, E)$. Then one has the following:

(1) $\widetilde{\bigwedge}_{i}\left(\Lambda_{i}, \Sigma_{i}\right)^{c} \subseteq\left(\widetilde{\nabla}_{i}\left(\Lambda_{i}, \Sigma_{i}\right)\right)^{c}$,

(2) $\left(\widetilde{\bigwedge}_{i}\left(\Lambda_{i}, \Sigma_{i}\right)\right)^{c} \subseteq \widetilde{\nabla}_{i}\left(\Lambda_{i}, \Sigma_{i}\right)^{c}$.

Finally, Theorem 3 may also be generalized. 
Theorem 5. Let $\mathbb{F}=\left\{\left(\Lambda_{i}, \Sigma\right) \mid i \in I\right\}$ be a family of fuzzy soft sets in a fuzzy soft class $(X, E)$. Then one has the following:

(1) $\widetilde{\bigwedge}_{i}\left(\Lambda_{i}, \Sigma\right)^{c}=\left(\widetilde{\nabla}_{i}\left(\Lambda_{i}, \Sigma\right)\right)^{c}$

(2) $\left(\widetilde{\bigwedge}_{i}\left(\Lambda_{i}, \Sigma\right)\right)^{c}=\widetilde{\nabla}_{i}\left(\Lambda_{i}, \Sigma\right)^{c}$.

\section{Conclusion}

The soft set theory proposed by Molodtsov offers a general mathematical tool for dealing with uncertain and vague objects. The researchers have contributed towards the fuzzification of Soft Set Theory. This paper contributes some more properties of fuzzy soft union and fuzzy soft intersection as defined and studied in [6-8] and supports them with examples and counterexamples. Arbitrary fuzzy soft union, arbitrary fuzzy soft intersection have been defined. DeMorgan Inclusions and DeMorgan Laws have also been given for an arbitrary collection of fuzzy soft sets. It is hoped that our findings will help enhancing this study on fuzzy soft sets for the researchers.

\section{Acknowledgment}

The authors gratefully acknowledge the comments of the referee which led to the improvment of this paper.

\section{References}

[1] D. Molodtsov, "Soft set theory-first results," Computers \& Mathematics with Applications, vol. 37, no. 4-5, pp. 19-31, 1999.

[2] P. K. Maji, A. R. Roy, and R. Biswas, "An application of soft sets in a decision making problem," Computers \& Mathematics with Applications, vol. 44, no. 8-9, pp. 1077-1083, 2002.

[3] P. K. Maji, R. Biswas, and A. R. Roy, "Soft set theory," Computers \& Mathematics with Applications, vol. 45, no. 4-5, pp. 555-562, 2003.

[4] D. Pei and D. Miao, "From soft sets to information systems," in Proceedings of the IEEE International Conference on Granular Computing, vol. 2, pp. 617-621, 2005.

[5] D. Chen, E. C. C. Tsang, D. S. Yeung, and X. Wang, "The parameterization reduction of soft sets and its applications," Computers \& Mathematics with Applications, vol. 49, no. 5-6, pp. 757-763, 2005.

[6] P. K. Maji, R. Biswas, and A. R. Roy, "Fuzzy soft sets," Journal of Fuzzy Mathematics, vol. 9, no. 3, pp. 589-602, 2001.

[7] A. R. Roy and P. K. Maji, "A fuzzy soft set theoretic approach to decision making problems," Journal of Computational and Applied Mathematics, vol. 203, no. 2, pp. 412-418, 2007.

[8] X. Yang, D. Yu, J. Yang, and C. Wu, "Generalization of soft set theory: from crisp to fuzzy case," in Proceedings of the 2nd International Conference of Fuzzy Information and Engineering (ICFIE '07), vol. 40 of Advances in Soft Computing, pp. 345354, 2007.

[9] T. Y. Lin, "A set theory for soft computing a unified view of fuzzy sets via neighborhoods," in Proceedings of the IEEE International Conference on Fuzzy Systems, vol. 2, pp. 1140 1146, New Orleans, La, USA, September 1996.
[10] A. Rosenfeld, "Fuzzy groups," Journal of Mathematical Analysis and Applications, vol. 35, pp. 512-517, 1971.

[11] Z. Xiao, L. Chen, B. Zhong, and S. Ye, "Recognition for soft information based on the theory of soft sets," in Proceedings of the International Conference on Services Systems and Services Management (ICSSSM '05), vol. 2, pp. 1104-1106, Chongquing, China, June 2005. 

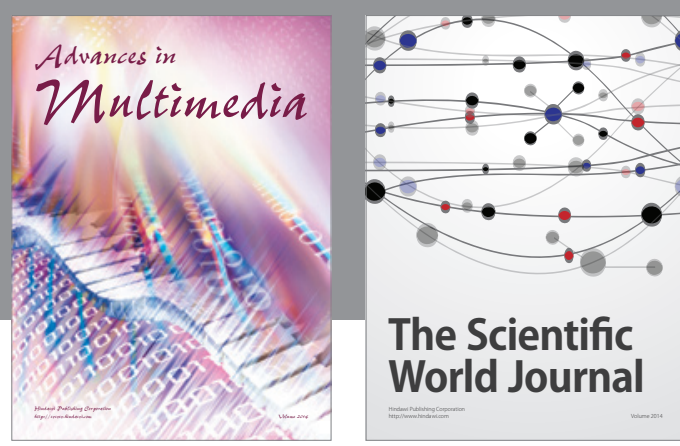

The Scientific World Journal
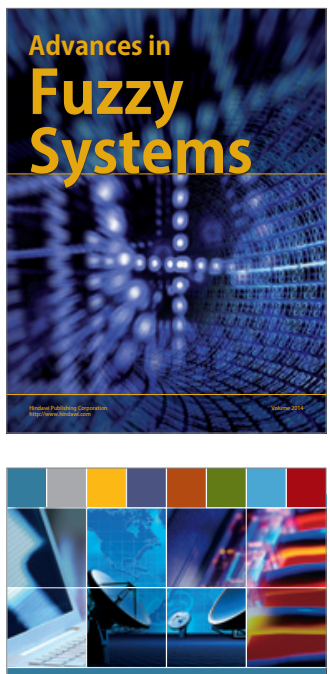

Computer Networks and Communications
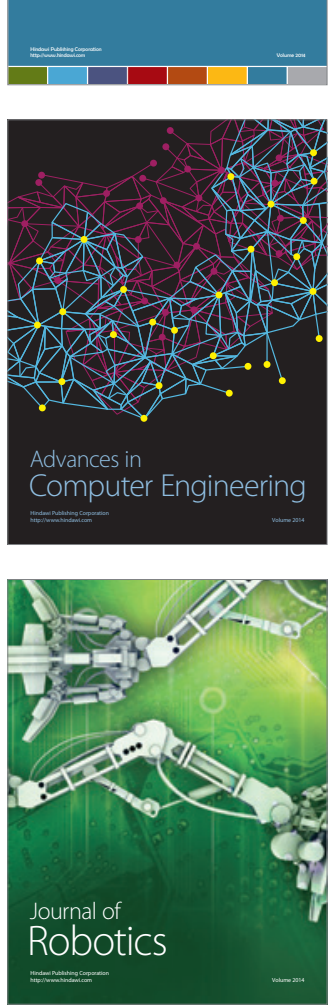
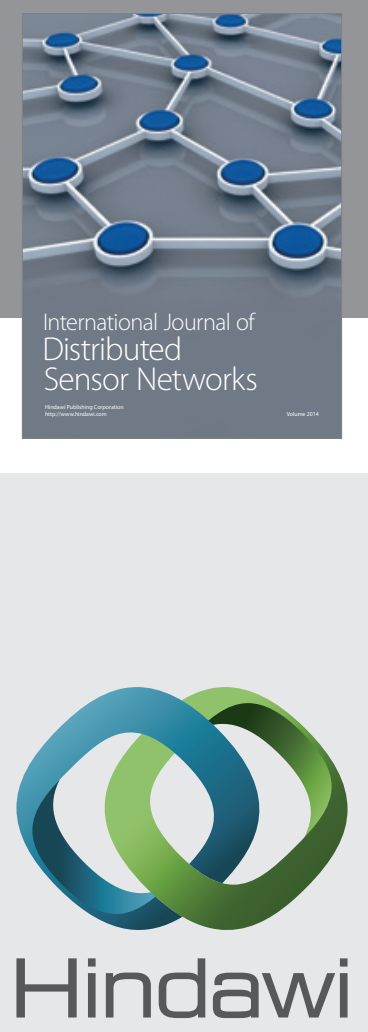

Submit your manuscripts at

http://www.hindawi.com
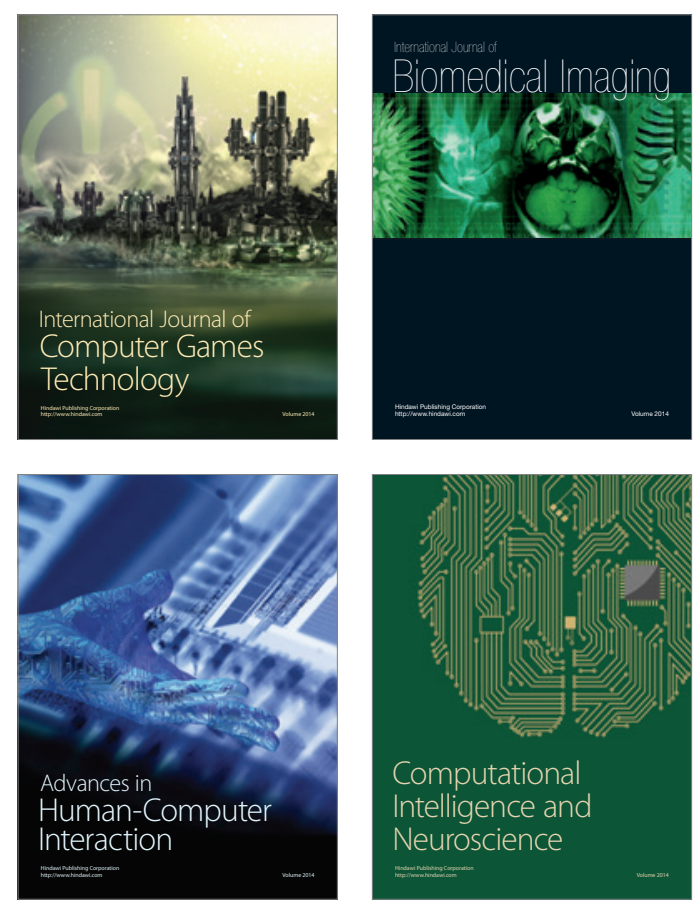
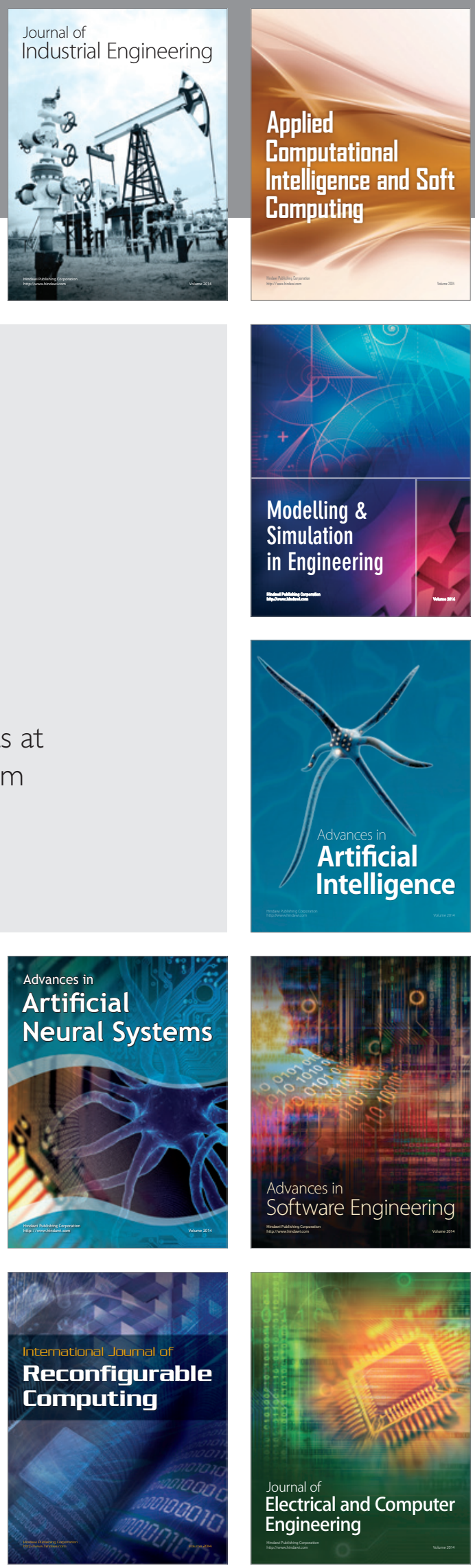